\title{
Fundamental Limits to Stereotactic Proton Therapy
}

\author{
Stephen Peggs \\ "This paper was submitted to the Conference Record for the \\ IEEE Nuclear Science Symposium/Medical Imaging Conference October 2003."
}

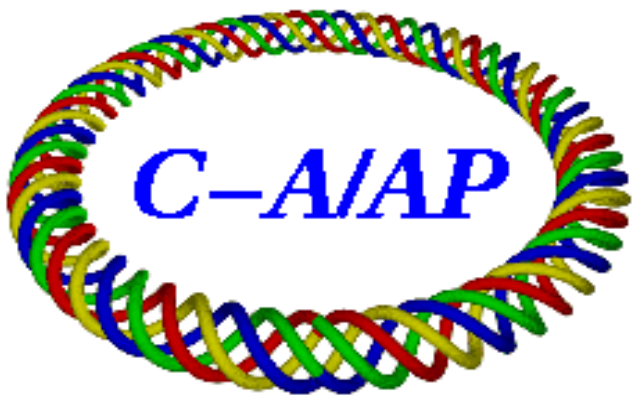

Collider-Accelerator Department Brookhaven National Laboratory

Upton, NY 11973 


\title{
Fundamental Limits to Stereotactic Proton Therapy
}

\author{
Stephen Peggs, Brookhaven National Laboratory, Upton NY 1973
}

\begin{abstract}
Proton therapy treatment techniques continue their steady development from passive scattering to 3-D multi-field scanning modalities. As the demands for faster and more accurate treatment increase, the requirements and tolerances on beam delivery technologies become more stringent. Fundamental physical limits (energy straggling and multiple Coulomb scattering) ultimately limit treatment performance parameters, even for an ideal accelerator and nozzle system. This paper calculates how few independent beam delivery control points are needed in a tumor in order to perform the sharpest possible stereotactic surgery, with $1 \%$ integrated dose flatness.
\end{abstract}

Index Terms-Proton therapy, dose delivery, gantry, nozzle, treatment planning.

\section{INTRODUCTION}

$\mathbf{M}$ OST contemporary proton therapy beamlines use passive scattering nozzles [1]. In this mode the incoming beam passes through a significant amount of matter in "beam spreaders", just before entering the patient. These spread the transverse profile of the beam, in order to deliver a broad and flat dose distribution to a tumor at the end of the proton beam range. They also inevitably increase the energy spread of the beam, resulting in a broader range distribution. Fixed energy proton sources (such as cyclotrons) also require "energy degraders" in the beam, to independently tune the average energy (and range) of the beam delivered to the patient.
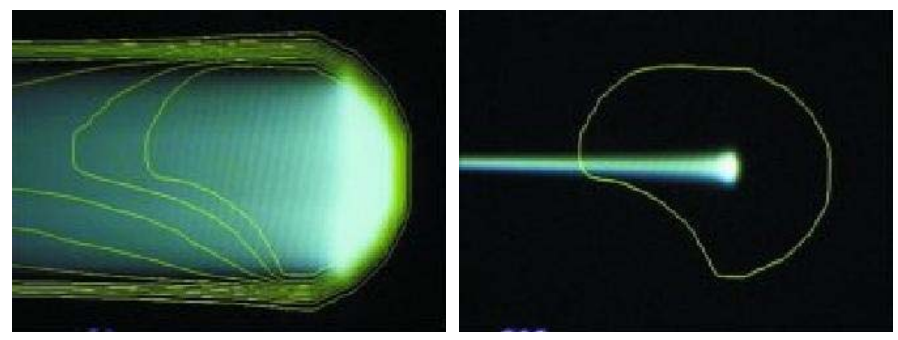

Fig. 1. Lateral scanning at multiple range layers (Pedroni et al). LEFT: The integrated dose is built up layer by layer. RIGHT: The fundamental physics of energy straggling and multiple Coulomb scattering blur the single beam delivery dose longitudinally and laterally.

Some advanced beamlines use active scanning techniques to deliver a radiation dose that conforms much more closely to the contours of the tumor. Figure 1 (LEFT) shows how the total dose to the patient is gradually built up in many "range layers", each corresponding to one particular average beam energy. The beam is scanned laterally in two dimensions by adjusting steering magnets, to best fit the outline of the tumor at that depth. Then the energy is reduced slightly, and the next energy layer is scanned. Conformal treatment succeeds because there is less material in the beamline - the lateral and longitudinal dose distributions are much narrower for each beam delivery. The oncologists "knife" is much sharper in active scanning, within limits that apply even for a perfect proton source.

This paper assumes a perfect incoming beam that has negligible energy spread and negligible emittance (lateral size), regardless of whether the proton source is a cyclotron or a synchrotron, extraction is fast or slow, et cetera. The sharpness of the knife is then limited by the physics of proton passage through matter. For example, Figure 1 (RIGHT) illustrates how energy straggling leads to a finite spread in range and a somewhat blurry Bragg peak for a single beam delivery, while multiple Coulomb scattering broadens the beam.

Active scanning can be performed in various ways. A continuous beam from a cyclotron, or slowly extracted from a synchrotron, may pause at a sequence of control points in each energy layer, quickly moving to the next point when enough local dose has been accumulated. Alternatively, fast extracted beam pulses can be delivered to each control point, one after the other. In many clinical cases the dose distribution is enhanced if beam is delivered to the same set of control points from more than one direction, or "field". Some facilities choose to pass over the same set of control points multiple times.

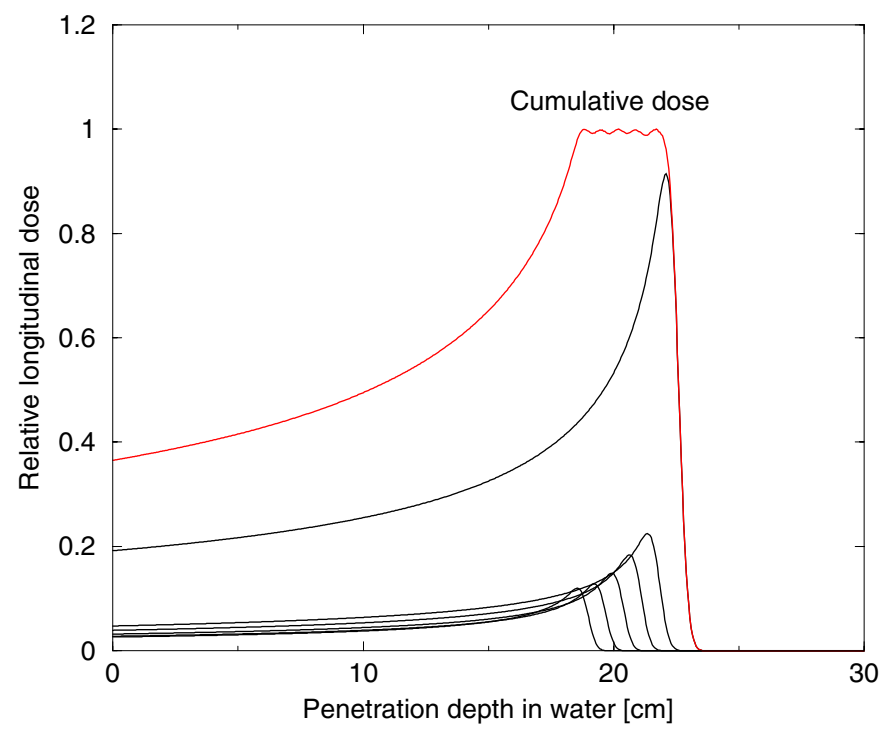

Fig. 2. Longitudinal dose versus depth, showing sharp Bragg peaks for each of 6 beam energies. After traversing about $20 \mathrm{~cm}$ of water the beam acquires an RMS energy width of about $1.4 \mathrm{MeV}$, due solely to energy straggling. Six beam pulses spaced in energy by $3.4 \mathrm{MeV}$ add to give a cumulative dose in a spread out Bragg peak that is flat at the $1 \%$ level.

This paper avoids such scanning implementation details by asking a simple question: "How few independent control points are needed in a tumor in order to perform the sharpest possible 


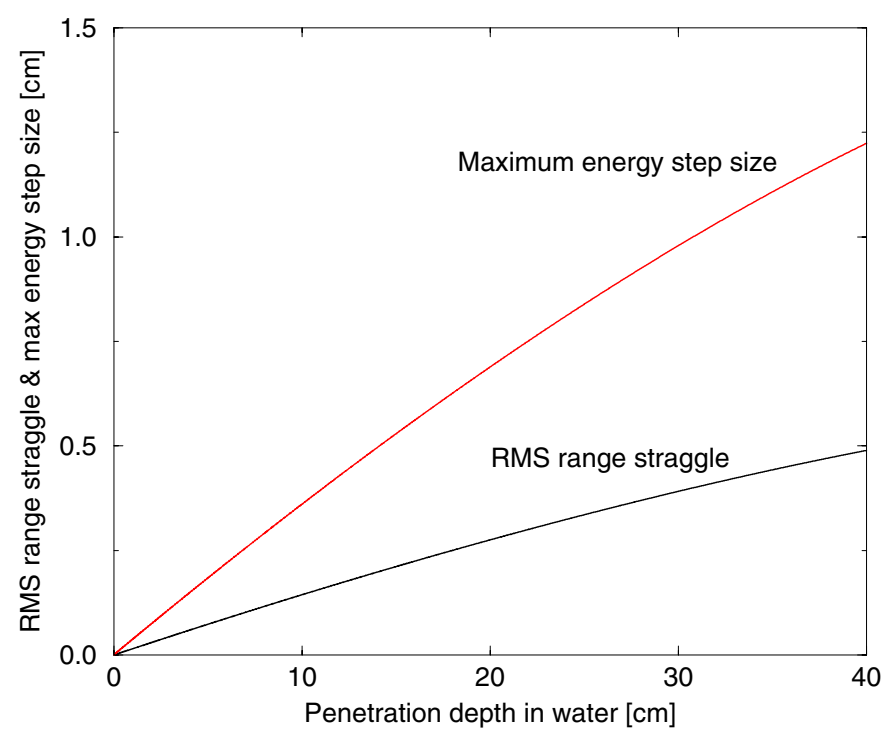

Fig. 3. Range straggling and the maximum spacing of energy layers. Because of the long dose tail upstream of the Bragg peak, it is possible to space the energy layers about 2.5 times further apart than the RMS range straggle, while maintaining $1 \%$ cumulative dose flatness.

stereotactic surgery (limited only by the physics of proton interactions with matter) with $1 \%$ integrated dose flatness?" An accurate answer to this question necessarily depends on the detailed properties of each individual tumor, and needs thorough application of a treatment planning system. The approximate answer given in this paper is nonetheless instructive, for example in the way that it scales with tumor volume for simple geometries.

\section{A. Straggling and multiple Coulomb scattering}

Protons deposit much of their energy and dose in a Bragg peak at the end of their range. The widths of the individual Bragg peaks in Figure 2 are due solely to energy straggling statistical fluctuations that accumulate as the protons traverse the patient, with no contribution from the energy spread of the incoming beam [2]. Figure 2 also shows how a longitudinal dose distribution with $1 \%$ flatness accumulates at a depth of about $20 \mathrm{~cm}$ in water when the beam energy is scanned in 3.4 MeV steps that are much larger than the RMS energy width of about $1.4 \mathrm{MeV}$. Figure 3 shows how RMS range straggle and maximum step size vary as function of penetration depth in water.

Similarly, multiple Coulomb scattering fluctuations cause the transverse beam size to grow and become significant, even for an ideal incoming beam with zero initial size. Figure 4 shows the evolution of the RMS transverse size of the beam, for a set of initial kinetic energies spaced by $50 \mathrm{MeV}$ [2].

\section{VARIABLE FOCUSING NOZZLE}

Figure 5 shows the transverse dose that accumulates when a sequence of Gaussian beam deliveries of varying size and

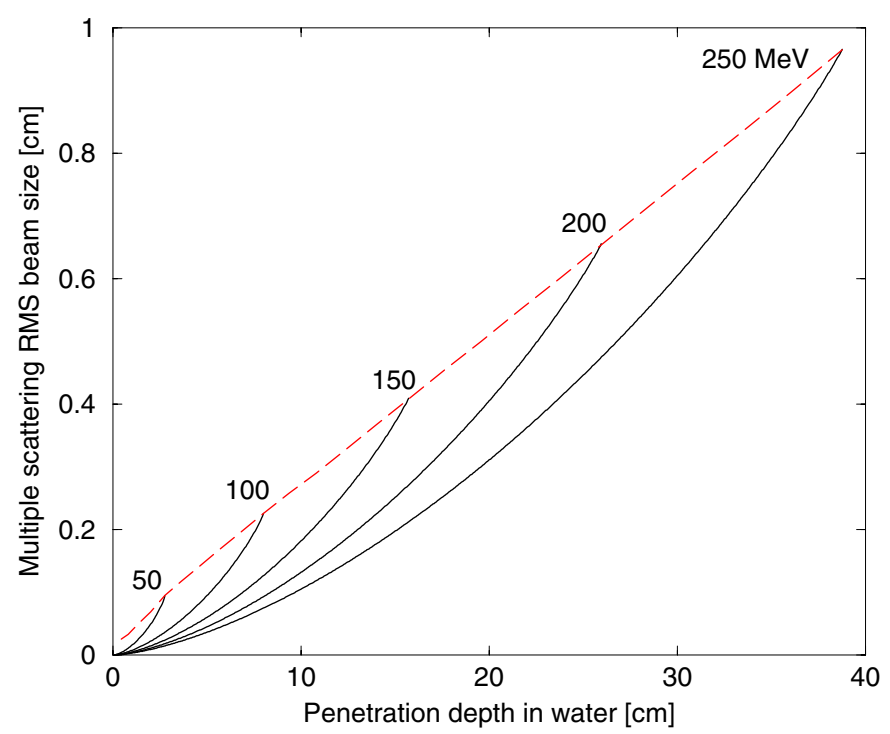

Fig. 4. Transverse proton beam size due to multiple Coulomb scattering. Although the beam enters with zero emittance, it acquires a near-Gaussian transverse beam distribution. For example, a beam with a range of about $20 \mathrm{~cm}$ accumulates an RMS size of $\sigma_{M S} \approx 0.5 \mathrm{~cm}$ by the end of its range.

intensity overlap. The spacing between the centers of deliveries $b$ and $b+1$ is set to be

$$
x_{b+1}-x_{b}=0.5\left(\sigma_{b+1}+\sigma_{b}\right)
$$

where $\sigma_{b}$ is the RMS size of the $b$ 'th delivery. This spacing ensures that the accumulated dose is flat to better than $1 \%$.

The total transverse beam size for each beam delivery, or pixel, is determined by two components that add in quadrature - the multiple scattering beam size $\sigma_{M S}$ and the optical beam size $\sigma_{O P T}-$ so that

$$
\sigma_{b}^{2}=\sigma_{M S}^{2}+\sigma_{O P T}^{2}
$$

The optical beam size

$$
\sigma_{O P T}^{2}=\epsilon \beta
$$

depends on both the unnormalized RMS emittance $\epsilon$ - the intrinsic size of the source beam - and the magnetic optical settings of the gantry and nozzle, represented by the "beta function" $\beta$ at the tumor.

Figure 6 shows two settings of a variable focusing nozzle that is capable of varying $\beta$ over more than a factor of 100 , corresponding to a dynamic range in the optical beam size $\sigma_{O P T}$ of more than 10. (This particular gantry and nozzle also provide a transverse scanning field of $\pm 20 \mathrm{~cm}$ [3].) It is necessary to be able to achieve

$$
\sigma_{O P T} \ll \sigma_{M S}
$$

in order to be able to achieve the smallest possible beam size. Thus the requirement on the beam delivery system is

$$
\epsilon \beta_{M I N} \ll \sigma_{M S}^{2}
$$

For example, if $\sigma_{M S}=0.5 \mathrm{~cm}$ and $\beta_{M I N}=1.0 \mathrm{~m}$, then it is necessary that $\epsilon \ll 25 \mu \mathrm{m}$. 


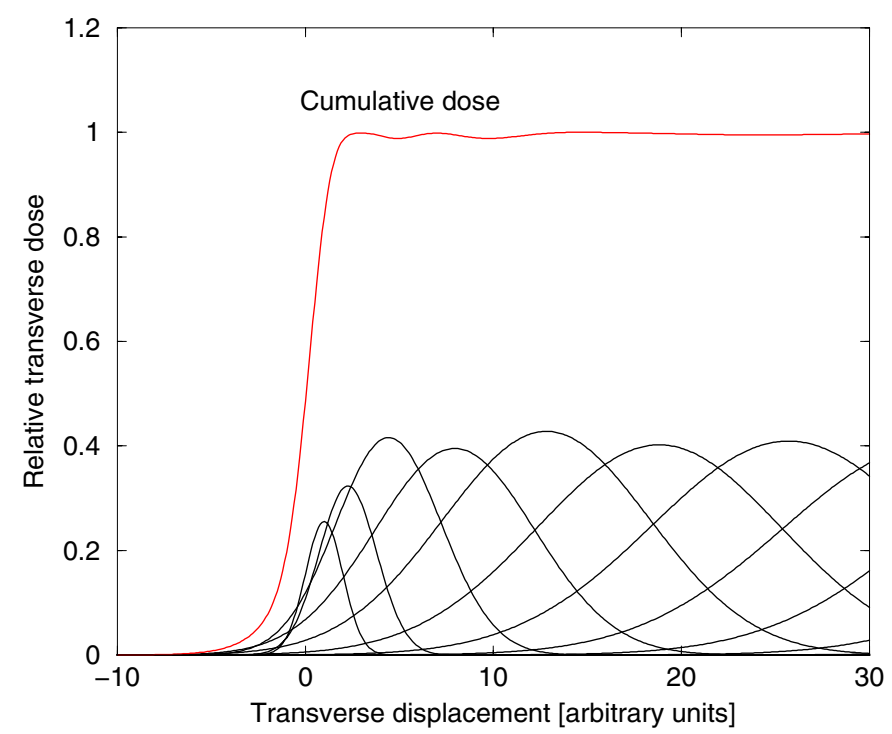

Fig. 5. Transverse dose profile from a sequence of overlapping Gaussian beam deliveries. The sharp edge is achieved by delivering a minimum width $\left(\sigma_{M S}\right)$ beam at the surface of the tumor. A cumulative dose flatnesses of $1 \%$ is achieved despite a beam size that increases rapidly as a function of depth.

\section{TUMOR PRISM MODEL}

Figure 7 shows a reasonably realistic model of a tumor that has the virtue of being straightforward to analyze. The tumor has the same cross section throughout its entire depth of distance $D-$ it is a prism. Different energy layers of the tumor are irradiated in sequence, by varying the energy of the incoming beam in steps. The area $A$ of each tumor layer and its perimeter length $C$ are conveniently associated through

$$
C=f \sqrt{4 \pi A}
$$

by introducing a form factor

$$
f \geq 1
$$

that measures the complexity of the convolutions around the perimeter of the layer, where the beam pixels are at their smallest. It attains the value of $f=1$ only for a perfect circle.

Figure 8 schematically represents a small section of the perimeter, showing how overlapping contours of constant size pixels are linked together. The pixel size increases for contours further from the edge of the layer, so that the total number of pixels within the "skin depth" is given by

$$
N_{S K I N}=\sum_{b=0}^{b_{S}} \frac{C}{\sigma_{b}}
$$

where $b_{S}$ is the number of the contour at which the pixel size effectively reaches its maximum. This can be rewritten as

$$
N_{S K I N}=\lambda_{S} \frac{C}{\sigma_{M S}}
$$

where the dimensionless "integrated linear density"

$$
\lambda_{S}=\sum_{b=0}^{b_{S}} \frac{\sigma_{M S}}{\sigma_{b}}
$$
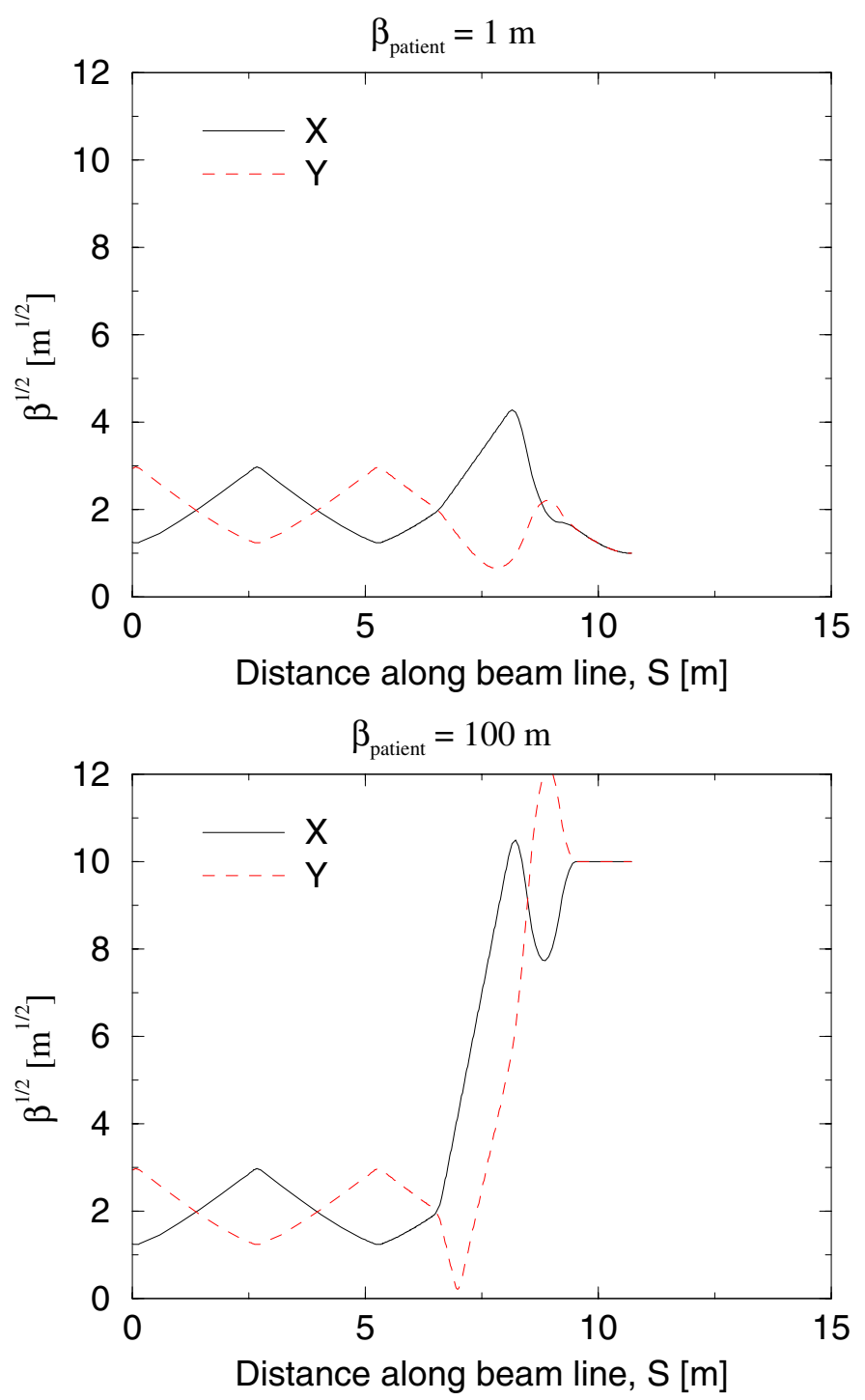

Fig. 6. Variable focusing nozzle optics can achieve a broad range of beam sizes at the patient. For example, varying $\beta$ from $1 \mathrm{~m}$ (TOP) to $100 \mathrm{~m}$ (BOTTOM) results in a dynamic range of 10 in the beam size component due to the beam delivery system. The total beam size is found by adding this component in quadrature with the multiple scattering size, $\sigma_{M S}$.

depends only on how fast the pixel sizes of successive contours converge to their final maximum value. The convenient form

$$
N_{S K I N} \approx\left(\lambda_{S} f \sqrt{4 \pi}\right) \frac{\sqrt{A}}{\sigma_{M S}}
$$

is found by substituting Equation 6 into Equation 9 .

In the rest of the layer, away from the skin depth, there are approximately

$$
N_{C O R E}=\frac{A}{M^{2} \sigma_{M S}^{2}}
$$

pixels, where $M$ is the dynamic range of the pixel size

$$
M \equiv \frac{\sigma_{M A X}}{\sigma_{M S}}
$$




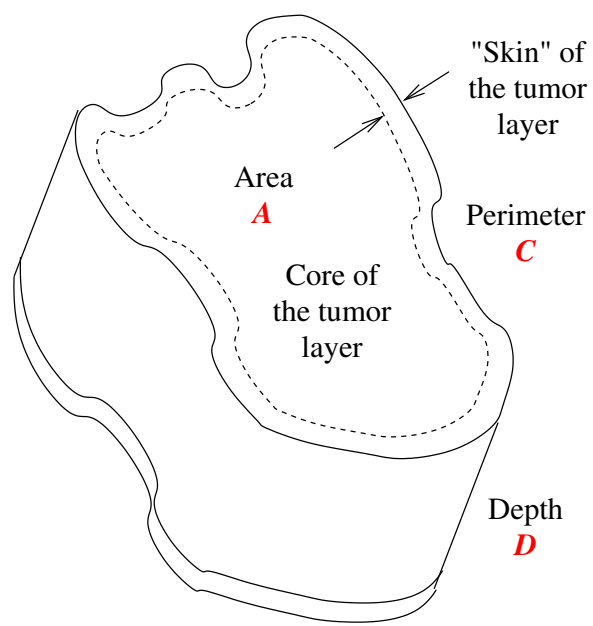

Fig. 7. Simple model of a tumor in the shape of a prism, with the same lateral cross section throughout the entire depth, $D$. The cross-sectional area is $A$, with a perimeter length $C$. The pixel size is significantly reduced inside the skin depth at the edge of each energy layer.

The total number of pixels per energy layer is dominated by the number of small pixels that are required to give a sharp edge around the perimeter, if

$$
N_{C O R E} \ll N_{S K I N}
$$

This condition is met if the dynamic range is large enough

$$
M^{2} \gg \frac{1}{\lambda_{S}} \frac{A}{C \sigma_{M S}}
$$

Substituting Equation 6 into Equation 15 gives the alternate convenient form

$$
M^{2} \gg\left(\frac{1}{\lambda_{S} f \sqrt{4 \pi}}\right) \frac{\sqrt{A}}{\sigma_{M S}}
$$

This condition is not hard to meet in practice, as will be seen below.

If the energy layers are uniformly spaced by $\Delta$, then about

$$
N_{E}=\frac{D}{\Delta}
$$

different energies are required to scan the entire depth of the tumor. Thus the grand total of the number of control points, or voxels, required to irradiate the tumor is about

$$
N_{T O T}=\lambda_{S} \frac{D C}{\Delta \sigma_{M S}}
$$

which can also be written

$$
N_{T O T} \approx\left(\lambda_{S} f \sqrt{4 \pi}\right) \frac{D \sqrt{A}}{\Delta \sigma_{M S}}
$$

in a more convenient form.

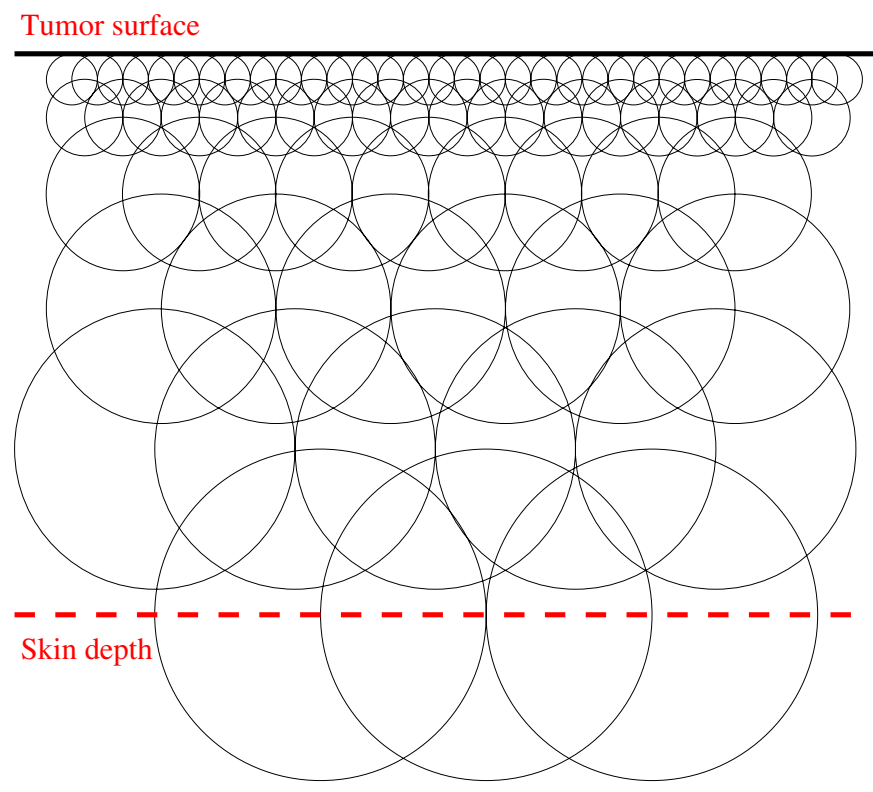

Fig. 8. Cartoon depiction of increasing pixel size at overlapping contours near the lateral edge - the tumor surface - of each energy layer. The pixel size is close to its asymptotic value at the contour with index number $b_{S}=5$, at the skin depth.

\section{A TYPICAL CONTOUR SIZE SERIES}

One of many plausible contour size series is

$$
\sigma_{b}=\sigma_{M S}\left(1+(M-1) \frac{\left(b / b_{0}\right)^{2}}{1+\left(b / b_{0}\right)^{2}}\right)
$$

as shown in Figure 9 (TOP). Figure 5 demonstrates that this series results in an accumulated dose profile with $1 \%$ flatness when the knee of the series $b_{0}=4$ and the dynamic range of the pixel size is $M=10$. In this case Figure 9 (MIDDLE) shows that the skin depth is effectively

$$
b_{S}=5
$$

resulting in an integrated linear density of

$$
\lambda_{S}=2.6
$$

Thus the approximate number of pixels in each energy layer is

$$
N_{S K I N} \approx 9.2 f \frac{\sqrt{A}}{\sigma_{M S}}
$$

while the condition for the number of pixels in the core to be negligible is

$$
M^{2} \gg \frac{0.11}{f} \frac{\sqrt{A}}{\sigma_{M S}}
$$

and the total number of voxels required is

$$
N_{T O T} \approx 9.2 f \frac{D \sqrt{A}}{\Delta \sigma_{M S}}
$$

It is interesting to evaluate these quantities in a numerical example. 

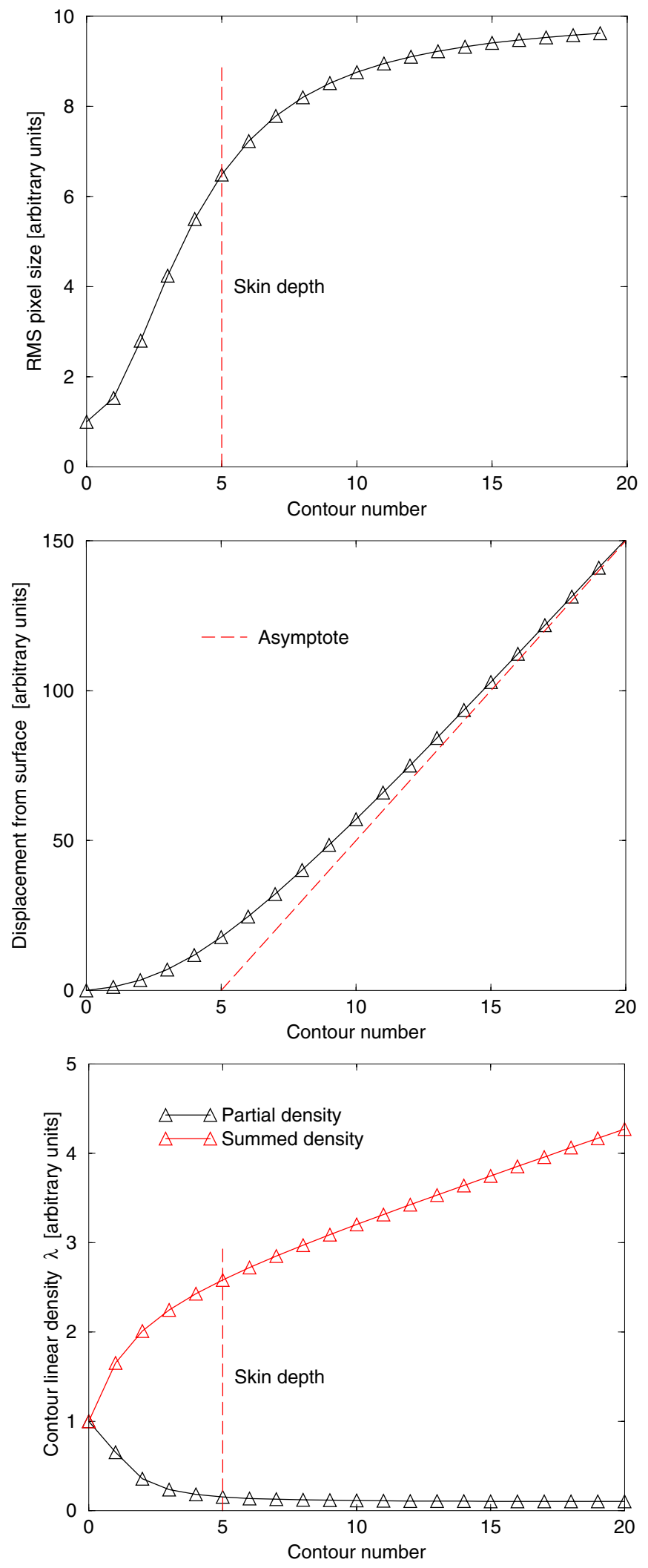

Fig. 9. Lateral contour distributions. TOP: Pixel size increases by a factor of 10 for deep contours. MIDDLE: The effective skin depth boundary is at contour $b_{S}=5$. BOTTOM: Linear density of pixels versus contour number The accumulated linear density is $\lambda_{S}=2.6$ at the skin depth.

\section{LARGE AND SMALL TUMORS}

Suppose that a tumor prism has a total depth $D=10 \mathrm{~cm}$ and a cross sectional area $A=100 \mathrm{~cm}^{2}$, for a total volume of 1 liter - this is an unusually large tumor. If the tumor is located at an average depth of about $20 \mathrm{~cm}$, then $\sigma_{M S} \approx 0.5 \mathrm{~cm}$, and

$$
N_{S K I N} \approx 184 \mathrm{f}
$$

The number of pixels in the core of each layer is insignificant by comparison to the number in the skin if

$$
M \gg \sqrt{\frac{2.2}{f}}
$$

showing that not much dynamic range is needed, even for the largest tumors. Figure 3 shows that it is possible to space the energy layers by about $\Delta=0.7 \mathrm{~cm}$ at an average depth of about $20 \mathrm{~cm}$, while still maintaining $1 \%$ cumulative dose flatness. Thus there are about $N_{E}=14$ energy layers, and about

$$
N_{T O T} \approx 2600 \mathrm{f}
$$

voxels, or control points, are needed in total.

If the height, width, and depth of the tumor scale together, then the number of voxels scales like

$$
N_{T O T} \sim V^{2 / 3}
$$

For example, a more typical tumor with a volume of $125 \mathrm{cc}$ requires approximately

$$
N_{T O T} \approx 650 \mathrm{f}
$$

voxels, or control points, in total.

\section{Conclusions}

For a simple model of a tumor shaped like a prism, about 1,000 independent control points are needed to perform the sharpest possible stereotactic surgery, limited only by the physics of proton interactions with matter, with $1 \%$ integrated dose flatness. This assumes that a modest adjustment of the lateral size of the beam at the tumor is possible, so that the edges of the tumor receive the sharpest possible beam distributions.

\section{ACKNOWLEDGMENT}

I would like to thank the members of the RCMS and PCT collaborations, T. Bohringer, A. Lomax, E. Pedroni, and U. Schneider, for their intellectual support and stimulating discussions.

\section{REFERENCES}

[1] M. Goitein, A. Lomax, E. Pedroni, Treating Cancer with Protons, Physics Today, September 2002.

[2] T. Satogata et al, Dose and Sensitivity in Proton Computed Tomography, These proceedings, 2003.

[3] C. Gardner, S. Peggs, (editors), Conceptual Design of the RCMS, Internal technical note, BNL, 2003. 